\title{
Nouveaux résultats d'estimation de l'héritabilité des gains en course des pur-sang en France
}

\author{
B Langlois, C Blouin, A Tavernier \\ Station de génétique quantitative et appliquée, \\ Institut national de la recherche agronomique, \\ 78352 Jouy-en-Josas cedex, France
}

(Reçu le 14 novembre 1994; accepté le 26 février 1996)

\begin{abstract}
Summary - New results on the estimation of the heritabilities of earnings of thoroughbreds in France. Logarithms of yearly earnings of 232893 horses in flat races and 85145 horses in steeplechases and hurdle races in France from 1950 to 1990 were analysed according to an animal model. REML methodology was applied to chronological subsamples of this enormous file to estimate genetic parameters. In flat races, from the birth year 1960 to 1987, heritability increased from 0.22 to 0.28 according to a statistically highly significant linear trend, and the repeatability between 2 and 3 years changed in a similary significant manner from 0.35 to 0.40 . Over the jumps, from 1962 to 1985, fluctuations appeared probably due to a lower precision of the estimates and no significant evolution could be seen for heritability (near 0.25). Repeatability between 4 and 5 years, however, increased significantly. The reasons for the evolution of these parameters over time are discussed. Two main hypotheses are mentioned: (i) introduction of external genetic variability in French pedigrees through American blood (this hypothesis however is not in agreement with the very small genetic distance between the two populations); and (ii) an equilibrium between two opposite sources generating linkage disequilibrium, selection and assortative mating. The intensity of these two forces observed in each subsample allows us to explain fairly well the variations of observed parameters.
\end{abstract}

thoroughbred / racing ability / genetic parameter / assortative mating

Résumé - Le logarithme du gain annuel de 232893 chevaux en plat et de 85145 chevaux sur les obstacles ayant couru en France au galop de 1950 à 1990 a été analysé selon un modèle animal. La méthode REML a permis d'estimer les composantes de la variance sur un ensemble de sous-fichiers établis en fonction de cohortes d'année de naissance. En plat, sur les naissances de 1960 à 1987, l'héritabilité a augmenté d'une manière hautement significative linéairement de 0,22 à 0,28 , tandis que la répétabilité des performances entre 2 et 3 ans connaissait une évolution très significative parallèle passant de 0,35 à 0,40 . En obstacles sur les naissances de 1962 à 1965, à cause des à-coups dus à la moindre précision des estimations, on ne perçoit pas d'évolution significative de l'héritabilité (autour de $0,25)$. La répétabilité entre 4 et 5 ans augmente cependant significativement. Les raisons 
de l'évolution de ces paramètres au cours du temps sont discutées. On retiendra deux hypothèses : i) un apport de variabilité extérieure par l'intermédiaire du sang américain dans les origines françaises, qui pourrait expliquer la variation des paramètres. Cette hypothèse se heurte cependant à la grande proximité génétique des deux populations; ii) un jeu d'équilibre entre deux sources principales de création de déséquilibre de liaison que sont la sélection et l'homogamie, dont l'intensité observée dans chaque sous échantillon permet d'expliquer assez bien les variations observées des paramètres.

pur-sang / aptitude au galop / paramètres génétiques / homogamie

\section{INTRODUCTION}

La population de pur-sang est connue pour être soumise de longue date et de façon intense aux effets conjoints de la sélection et de l'homogamie. La variabilité génétique que l'on peut y mesurer a donc deux composantes : une qui résulte de la variabilité des effets des gènes et qui est celle que l'on pourrait observer en panmixie, l'autre qui résulte de déséquilibres de liaison induits par les écarts à la panmixie précités. Si la première composante dans l'hypothèse d'un grand nombre de gènes a la réputation de varier fort peu d'une génération à la suivante, la seconde en revanche peut évoluer rapidement jusqu'à l'obtention d'un état d'équilibre stable (Verrier et al, 1991). On peut donc se demander où nous en sommes vis-à-vis de ces phénomènes pour les populations que nous avons à traiter. Pouvons-nous compter sur des paramètres génétiques stables ou bien au contraire évoluent-ils encore? C'est cette question que nous nous proposons d'aborder ici.

\section{MATÉRIEL ET MÉTHODE}

\section{Les données}

Les gains annuels en courses plates et à obstacles en France de 1950 à 1993 ont été informatisés à partir des documents publiés - Anonyme (1950-1968) et Anonyme (1969-1982). Depuis 1983, ils nous ont été fournis par le GIE France Galop.

De cette masse très importante de données vérifiées avec la base généalogique nationale du Sire, nous avons extrait un certain nombre de sous-fichiers pour procéder à des estimations des paramètres génétiques.

La technique de constitution de ces sous-fichiers est la suivante :

i) Nous ne conservons que les gains à 2 et 3 ans en plat et à 4 et 5 ans en obstacles.

ii) Nous sélectionnons des cohortes de quatre années de naissance. Les parents de ces chevaux constituent la généalogie utilisée dans l'analyse. L'origine de tous les autres chevaux présents dans le fichier est mise à zéro.

iii) Nous ne retenons que les performances dans l'intervalle année de naissance moins onze ans et année de naissance plus trois ans pour le plat, et année de naissance moins neuf ans et année de naissance plus cinq ans pour l'obstacle. 
Nous pouvons ainsi constituer sept sous-fichiers analogues pour le plat et six pour l'obstacle, où nous sommes sûrs de disposer d'un maximum de relations parent-descendant tout en ayant des descendances suffisamment nombreuses par reproducteur.

Les caractéristiques de ces sous-fichiers sont fournies aux tableaux I, II et III.

Tableau I. Règles de constitution des sous-fichiers.

Plat à 2 et 3 ans

\begin{tabular}{ccccc}
\hline $\begin{array}{c}\text { Année de } \\
\text { naissance de } \\
\text { référence pour la } \\
\text { définition des } \\
\text { cohortes }\end{array}$ & $\begin{array}{c}\text { Année de } \\
\text { performances } \\
\text { considérée }\end{array}$ & & $\begin{array}{c}\text { Année de } \\
\text { naissance de } \\
\text { référence pour la } \\
\text { définition des } \\
\text { cohortes }\end{array}$ & $\begin{array}{c}\text { Année de } \\
\text { performances } \\
\text { considérée }\end{array}$ \\
\hline $60 \longrightarrow 63$ & $50 \longrightarrow 66$ & & $62 \longrightarrow 65$ & \\
$64 \longrightarrow 67$ & $53 \longrightarrow 70$ & & $66 \longrightarrow 69$ & $53 \longrightarrow 70$ \\
$68 \longrightarrow 71$ & $57 \longrightarrow 74$ & & $70 \longrightarrow 73$ & $57 \longrightarrow 74$ \\
$72 \longrightarrow 75$ & $61 \longrightarrow 78$ & & $74 \longrightarrow 77$ & $61 \longrightarrow 78$ \\
$76 \longrightarrow 79$ & $65 \longrightarrow 82$ & & $78 \longrightarrow 81$ & $65 \longrightarrow 82$ \\
$80 \longrightarrow 83$ & $69 \longrightarrow 86$ & $82 \longrightarrow 85$ & $69 \longrightarrow 86$ \\
$84 \longrightarrow 87$ & $73 \longrightarrow 90$ & & $73 \longrightarrow 90$ \\
\hline
\end{tabular}

Tableau II. Principales caractéristiques des sous-fichiers concernant le plat.

\begin{tabular}{|c|c|c|c|c|c|c|c|}
\hline $\begin{array}{l}\text { Fichiers des naissances } \\
\text { Nombre de chevaux }\end{array}$ & $\begin{array}{l}60-63 \\
20660\end{array}$ & $\begin{array}{l}64-67 \\
25679\end{array}$ & $\begin{array}{r}68-71 \\
30585\end{array}$ & $\begin{array}{l}72-75 \\
35299\end{array}$ & $\begin{array}{r}76-79 \\
37677\end{array}$ & $\begin{array}{l}80-83 \\
40710\end{array}$ & $\begin{array}{l}84-87 \\
42283\end{array}$ \\
\hline $\begin{array}{l}\text { Nombre moyen d'années } \\
\text { de performances / cheval }\end{array}$ & 1,40 & 1,38 & 1,37 & 1,37 & 1,37 & 1,36 & 1,34 \\
\hline Nombre de pères & 366 & 619 & 822 & 941 & 844 & 1010 & 952 \\
\hline $\begin{array}{l}\text { Nombre moyen } \\
\text { de descendants / père }\end{array}$ & 11,04 & 10,20 & 9,73 & 9,28 & 9,57 & 8,67 & 9,68 \\
\hline Nombre de mères & 2942 & 4488 & 5679 & 6109 & 5498 & 6139 & 6463 \\
\hline $\begin{array}{l}\text { Nombre moyen } \\
\text { de descendants / mère }\end{array}$ & 1,53 & 1,47 & 1,43 & 1,43 & 1,47 & 1,43 & 1,43 \\
\hline $\begin{array}{l}\text { Nombre de couples } \\
\text { père-descendants }\end{array}$ & 3485 & 4407 & 4899 & 5004 & 4829 & 5142 & 5714 \\
\hline $\begin{array}{l}\text { Nombre de couples } \\
\text { mère-descendants }\end{array}$ & 2804 & 3653 & 4130 & 4412 & 4250 & 4172 & 4352 \\
\hline
\end{tabular}


Tableau III. Principales caractéristiques des sous-fichiers concernant l'obstacle.

\begin{tabular}{|c|c|c|c|c|c|c|}
\hline $\begin{array}{l}\text { Fichiers des naissances } \\
\text { Nombre de chevaux }\end{array}$ & $\begin{array}{l}62-65 \\
9784\end{array}$ & $\begin{array}{l}66-69 \\
11870\end{array}$ & $\begin{array}{l}70-73 \\
13791\end{array}$ & $\begin{array}{l}74-77 \\
15024\end{array}$ & $\begin{array}{l}78-81 \\
16765\end{array}$ & $\begin{array}{l}82-85 \\
17911\end{array}$ \\
\hline $\begin{array}{l}\text { Nombre moyen d'années } \\
\text { de performances/cheval }\end{array}$ & 1,37 & 1,36 & 1,36 & 1,36 & 1,35 & 1,34 \\
\hline Nombre de pères & 365 & 535 & 678 & 630 & 713 & 710 \\
\hline $\begin{array}{l}\text { Nombre moyen } \\
\text { de descendants/père }\end{array}$ & 5,38 & 5,02 & 4,60 & 4,66 & 4,84 & 5,10 \\
\hline Nombre de mères & 1964 & 2686 & 3118 & 2934 & 3448 & 3623 \\
\hline $\begin{array}{l}\text { Nombre moyen } \\
\text { de descendants/mère }\end{array}$ & 1,22 & 1,19 & 1,19 & 1,19 & 1,22 & 1,22 \\
\hline Nombre de couples père-descendants & 100 & 158 & 216 & 151 & 276 & 303 \\
\hline Nombre de couples mère-descendants & 349 & 425 & 412 & 435 & 483 & 540 \\
\hline
\end{tabular}

\section{Les analyses}

Pour chaque sous-fichier, le modèle animal suivant selon Tavernier (1988) a permis d'estimer par un REML, grâce à l'algorithme EM, les composantes de la variance :

$$
\mathbf{y}=\mathbf{X b}+\mathbf{Z u}+\mathbf{W} \mathbf{m}+\mathbf{Z} \mathbf{p}+\mathbf{e}
$$

avec $\mathbf{y}$ : vecteur des logarithmes des gains annuels; $\mathbf{b}$ : vecteur des effets fixes : le sexe (deux niveaux : mâles et hongres, femelles), l'âge-année (deux catégories d'âges combinées avec 17 années en plat et 18 en obstacles, soit respectivement 34 et 36 effets); $\mathbf{u}$ : vecteur des valeurs génétiques additives; $\mathbf{m}$ : vecteur des effets de milieu commun aux différents descendants d'une même mère (effet maternelélevage); $\mathbf{p}$ : effets de milieu commun aux différentes performances d'un même cheval, hors l'effet maternel-élevage précité; $\mathbf{e}$ : vecteur des effets résiduels; $\mathbf{X}, \mathbf{Z}$ et $\mathbf{W}$ : matrices d'incidence correspondantes.

La matrice de variance-covariance entre les valeurs génétiques additives est la matrice de parenté. Tous les effets sont absorbés dans la matrice «effets fixes + valeur génétique des étalons». Cette matrice est alors inversée et les solutions et les autres inverses sont obtenues en réutilisant les équations. La convergence étant lente (l'écart entre deux estimations des composantes se situe en moyenne entre $10^{-3}$ et $10^{-4}$ ), on a procédé par approximations successives jusqu'à encadrer les valeurs finales. Selon les fichiers et les valeurs de départ, le nombre d'itérations a varié entre 60 et 120.

\section{RÉSULTATS}

Le tableau IV récapitule les résultats obtenus. En plat, en 27 ans, l'héritabilité a augmenté progressivement de 0,22 à 0,28 tandis que la répétabilité des performances entre 2 et 3 ans connaissait une évolution strictement parallèle passant de 0,35 à 
0,40. Sur la période, les effets maternel-élevage sont stables entre 0,05 et 0,06 . Il en est de même pour les effets de milieu permanent autres que maternel qui sont compris entre 0,07 et 0,08 .

Tableau IV. Résultats des estimations des paramètres génétiques.

Logarithme du gain annuel en courses plates

\begin{tabular}{lccccccc}
\hline Fichiers des naissances & $60-63$ & $64-67$ & $68-71$ & $72-75$ & $76-79$ & $80-83$ & $84-87$ \\
Répétabilité $\left(\sigma_{a}^{2}+\sigma_{m}^{2}+\sigma_{p}^{2}\right) / \sigma_{t}^{2}$ & 0,348 & 0,350 & 0,368 & 0,386 & 0,387 & 0,400 & 0,397 \\
Effet maternel - élevage $\sigma_{m}^{2} / \sigma_{t}^{2}$ & 0,056 & 0,054 & 0,058 & 0,058 & 0,058 & 0,046 & 0,045 \\
Héritabilité $\sigma_{a}^{2} / \sigma_{t}^{2}$ & 0,218 & 0,218 & 0,233 & 0,258 & 0,262 & 0,279 & 0,275 \\
Résiduelle $\sigma_{e}^{2}$ & 1,444 & 1,392 & 1,301 & 1,217 & 1,177 & 1,128 & 1,116 \\
Variance phénotypique $\sigma_{t}^{2} / \sigma_{e}^{2}$ & 1,534 & 1,538 & 1,583 & 1,628 & 1,631 & 1,667 & 1,659
\end{tabular}

Logarithme du gain annuel en courses à obstacles

\begin{tabular}{|c|c|c|c|c|c|c|}
\hline Fichiers des naissances & $62-65$ & $66-69$ & $70-73$ & $74-77$ & $78-81$ & $82-85$ \\
\hline Répétabilité $\left(\sigma_{a}^{2}+\sigma_{m}^{2}+\sigma_{p}^{2}\right) / \sigma_{t}^{2}$ & 0,410 & 0,445 & 0,432 & 0,441 & 0,441 & 0,451 \\
\hline Effet maternel - élevage $\sigma_{m}^{2} / \sigma_{t}^{2}$ & 0,055 & 0,061 & 0,054 & 0,059 & 0,064 & 0,061 \\
\hline Héritabilité $\sigma_{a}^{2} / \sigma_{t}^{2}$ & 0,214 & 0,261 & 0,257 & 0,255 & 0,242 & 0,274 \\
\hline Résiduelle $\sigma_{e}^{2}$ & 1,274 & 1,201 & 1,234 & 1,199 & 1,170 & 1,141 \\
\hline Variance phénotypique $\sigma_{t}^{2} / \sigma_{e}^{2}$ & 1,695 & 1,802 & 1,761 & 1,789 & 1,789 & 1,821 \\
\hline
\end{tabular}

L'ajustement linéaire de l'évolution de l'héritabilité $\left(h^{2}\right)$ et de la répétabilité $(r)$ en fonction de l'année de naissance donne une pente de régression $b=$ $0,0029 \pm 0,0009$ par an pour $h^{2}$ et $b^{\prime}=0,0024 \pm 0,0002$ par an pour $r$. Le test de linéarité de ces régressions a été réalisé en prenant des valeurs d'écart type d'estimation $\sigma\left(h^{2}\right)=0,02$ et $\sigma(r)=0,005$. Ces deux coefficients de régression sont significatifs au seuil de $1 \%$ et ne diffèrent pas significativement entre eux.

En obstacles, à cause des à-coups dus sans doute à la moindre précision des estimations, on ne perçoit pas une évolution très nette de l'héritabilité (autour de $0,25)$. Les effets «maternel-élevage» sont stables et se situent aussi entre 0,05 et 0,06 . Les effets de milieu permanent sont plus variables et se situent entre 0,14 et 0,12 . L'ajustement linéaire fournit une pente de régression $b=0,0017 \pm 0,0030$ pour $h^{2}$ et $b^{\prime}=0,0014 \pm 0,0006$ pour $r$. Le test de linéarité de ces régressions a été réalisé en prenant des valeurs d'écart type d'estimation $\sigma\left(h^{2}\right)=0,05$ et $\sigma(r)=0,01$. Seule l'évolution de la répétabilité est significative au seuil de $5 \%$. Ces deux coefficients de régression ne diffèrent pas significativement entre eux. Le parallélisme de l'évolution de $h^{2}$ et de $r$ en plat $(0,0029=0,0024)$ et en obstacles $(0,0017=0,0014)$ ne peut être exclu même en prenant des risques allant jusqu'à $50 \%$. Il ne paraît donc pas y avoir d'évolution des composantes maternel-élevage et milieu permanent dans les deux cas d'évaluation. 
L'examen des estimations des valeurs génétiques individuelles obtenues pour chacun des sous-échantillons étudiés permet de se faire une idée de l'intensité de la sélection, en comparant les valeurs des parents à celles de leurs descendants. De même la corrélation entre les estimations des parents accouplés permet d'apprécier l'intensité de l'homogamie. Le tableau $\mathrm{V}$ a été établi pour caractériser ces écarts à la panmixie et les mettre éventuellement en relation avec les paramètres trouvés.

Table V. Statistiques sur les Blup des chevaux dans chaque sous-fichier.

Courses plates à 2 et 3 ans

\begin{tabular}{lccccccc}
\hline Fichiers des naissances & $60-63$ & $64-67$ & $68-71$ & $72-75$ & $76-79$ & $80-83$ & $84-87$ \\
$\begin{array}{l}\text { Écart parent moyen-descendant } \\
\text { (en log de gain corrigé) }\end{array}$ & 0,28 & 0,20 & 0,19 & 0,20 & 0,24 & 0,26 & 0,26 \\
Valeur initiale 0, 28 = 100 & 100 & 71 & 68 & 71 & 86 & 93 & 93 \\
Corrélation père-mère & 0,21 & 0,26 & 0,29 & 0,27 & 0,31 & 0,29 & 0,29 \\
Rappel $h^{2}$ & 0,22 & 0,22 & 0,23 & 0,26 & 0,26 & 0,28 & 0,28 \\
\hline
\end{tabular}

Courses à obstacles à 4 et 5 ans

\begin{tabular}{lcccccc}
\hline Fichiers des naissances & $62-65$ & $66-69$ & $70-73$ & $74-77$ & $78-81$ & $82-85$ \\
Écart parent moyen - descendant & 0,04 & 0,05 & 0,04 & 0,04 & 0,04 & 0,07 \\
(en log de gain corrigé) & 14 & 18 & 14 & 14 & 14 & 25 \\
Valeur initiale 0, 28 = 100 & 0,18 & 0,21 & 0,23 & 0,25 & 0,23 & 0,21 \\
Corrélation père-mère & 0,21 & 0,26 & 0,26 & 0,26 & 0,24 & 0,27 \\
Rappel $h^{2}$ &
\end{tabular}

\section{DISCUSSION}

Les résultats obtenus en plat peuvent paraître paradoxaux puisque la variabilité génétique mise en évidence par ces différentes analyses en modèle animal augmente au cours des années alors que l'a priori général milite plutôt en faveur d'une diminution.

Bien que la précision de ces estimations n'ait pas été calculée, on peut essayer de s'en faire une idée à l'aide des tableaux II et III en faisant usage des formules décrites par Falconer (1981) pour l'écart type de l'héritabilité estimée par les relations père-descendant, mère-descendant et demi-germains paternels. En qualifiant de précision l'inverse de la variance d'erreur dans chaque cas et en postulant que la précision d'une estimation conjointe est égale à la somme des précisions des parties, ce qui postule l'indépendance des trois modes d'estimation proposés, on peut calculer un ordre de grandeur de l'écart type de l'héritabilité. On constate ainsi qu'il varie peu d'un échantillon à l'autre et qu'il se situe vers $\sigma\left(h^{2}\right) \approx 0,02$ pour le plat et $\sigma\left(h^{2}\right) \approx 0,05$ pour l'obstacle. Ce calcul approximatif, soumis à l'hypothèse d'indépendance des trois méthodes classiques, ne tient pas compte des 
autres relations de parenté utilisées par le modèle animal, c'est donc un écart type maximum si les trois voies sont réellement indépendantes.

Dans une approche classique d'additivité des effets du génotype et de l'environnement, cette augmentation peut être attribuée soit à une réduction de la variabilité des effets non contrôlés du milieu, soit à une croissance de la variabilité génétique. Pour éliminer les fluctuations de variance liées, non pas à la valeur des chevaux mais au système de dotation, nous fournissons au tableau IV la variance phénotypique rapportée à la variance résiduelle, qui exprime la variabilité entre chevaux à système de dotation normalisé. Il en résulte que, si la variance résiduelle paraît bien diminuer en valeur absolue au cours du temps en plat, il apparaît aussi que la variance phénotypique propre aux chevaux, c'est-à-dire à système de dotation standardisé $\left(\sigma_{e}^{2}=1\right)$, augmente en raison principalement de l'augmentation de la variabilité génétique puisque les autres composantes évoluent peu. Il semble donc bien que l'on ait affaire à une véritable augmentation de la variabilité génétique, qui entraîne à la fois l'augmentation de l'héritabilité et de la répétabilité.

À quoi serait-elle due? Deux raisons peuvent être invoquées :

i) Un apport de variabilité extérieure, par l'intermédiaire du sang américain dans les origines françaises.

ii) Une pratique intensive d'accouplements homogames, dont les effets d'étalement de la variabilité génétique ne seraient pas compensés par les effets de la sélection (Langlois, 1990).

Dans une approche moins classique, une autre raison pourrait être invoquée. Dans l'hypothèse de l'existence d'une corrélation génotype-milieu positive, nous savons que l'héritabilité mesurée est surévaluée (Langlois, 1981). Une modification progressive des conditions d'accès aux différents niveaux de course sur la période pourrait avoir entraîné une élévation de cette corrélation en prédéterminant de plus en plus la carrière d'un cheval en fonction de son origine. Le mécanisme par lequel s'installe cette corrélation dépend en particulier des conditions d'accès aux courses de maiden qui ouvrent la carrière classique. C'est un secteur réservé des grandes écuries et tout intrus s'y trouve défavorisé, car ces écuries s'arrangent pour lui opposer de très bons chevaux. En outre, si cet intrus n'arrive pas à se classer, il repart dans un circuit de courses plus modestes avec le désavantage d'une forte valeur handicap. Cela dissuade très souvent les petits d'affronter les grands et, bien que l'accès soit ouvert, cela conduit sans doute à favoriser les chevaux des grandes écuries. Toutefois le phénomène a toujours existé et ne paraît pas s'être intensifié sur la période. Remarquons en outre que le choix de la variable gain annuel a justement été fait pour réduire au maximum ce type de perturbations, les variables gain moyen annuel par départ ou par classement, réputées bien plus héritables (Hintz, 1977; Langlois, 1975, 1980; Tolley et al, 1985), étant plus suspectes de ce point de vue. Cette hypothèse d'évolution de la corrélation génotype milieu ne paraît donc pas très convaincante.

L'introduction d'une nouvelle variabilité génétique par l'usage du pur-sang américain ne nous paraît pas non plus devoir être retenue. En effet, comme en témoignent les études de distances génétiques réalisées dernièrement (Moureaux, 1994; Moureaux et al, 1996), les populations de la France et des Etats-Unis apparaissent extrêmement proches. De plus, si des différences de patrimoine génétique 
importantes entre les deux populations existaient, pourquoi l'effet serait-il perçu au niveau des performances en plat mais pas de façon aussi nette en obstacles?

En revanche, l'examen du tableau $\mathrm{V}$ permet d'interpréter en termes de déséquilibre de liaison les variations observées des paramètres génétiques de manière assez satisfaisante.

En plat, l'échantillon des naissances 60-63 correspond à la plus forte intensité de sélection et à la valeur la plus basse de la corrélation des valeurs génétiques entre conjoints. La sélection domine sur l'homogamie, l'héritabilité est alors à son point le plus bas. Plus tard, pour les naissances 64-67, la sélection se relâche quelque peu et l'homogamie augmente mais sans doute pas assez pour faire augmenter l'héritabilité. Cela ne se produit que sur les échantillons des naissances $68-71$ et 72-75. La sélection restant d'une intensité modérée, l'élévation du niveau d'homogamie permet de faire passer l'héritabilité à 0,23 puis 0,26 . À partir des naissances 76-79, la reprise progressive d'une forte intensité de sélection freine ce phénomène malgré la progression de l'homogamie, jusqu'aux naissances $80-83$ et 84-87 qui semblent marquer un nouveau point d'équilibre, la sélection et l'homogamie, toutes deux à un niveau haut, paraissant s'équilibrer.

En obstacles, la sélection est de bien moindre intensité mais la pratique de l'homogamie paraît exister quand même. Notons dans ce cas que le point bas de l'héritabilité, qui correspond aux naissances $62-65$, correspond aussi au point bas de la corrélation père-mère. Il est en outre difficile de conclure sur les variations des autres échantillons qui peuvent très bien être des fluctuations aléatoires dues à des effectifs beaucoup plus réduits qu'en plat.

Nous retiendrons en conclusion que la stabilité des paramètres génétiques généralement acceptée pour l'indexation des reproducteurs est une hypothèse qu'il faut manier avec précaution dans le cas des performances en plat des pur-sang.

Nous remarquerons toutefois que la moyenne des estimations de l'héritabilité de l'aptitude au galop mesurée par le gain annuel s'établit à la même valeur $h^{2}=0,25$ en plat comme en obstacles, avec une répétabilité moyenne de 0,38 entre 2 et 3 ans pour le plat, contre 0,44 entre 4 et 5 ans pour l'obstacle.

Les raisons de l'éventuelle sur- ou sous-évaluation de ces paramètres par rapport à leur valeur en panmixie nous paraissent devoir être recherchées dans la création de déséquilibres de liaison par l'homogamie et la sélection. On peut s'étonner que des phénomènes générateurs de déséquilibres de liaison décrits depuis aussi longtemps que l'homogamie (Fisher, 1918) ou la sélection (Lush, 1945; Bulmer, 1971) retiennent si peu l'attention des généticiens appliqués. Peu d'études relatent la variation de paramètres génétiques au cours du temps. De plus, la majorité de celles qui s'y consacrent traitent de la limite de la sélection par épuisement de la variabilité allélique. Citons, de façon non exhaustive, Wilson et al (1971) chez la souris, Yoo (1980) chez la drosophile, Hunton (1984) chez la volaille, Fredeen (1984) chez le porc, Mirande et Van Vleck (1985) chez la vache laitière; certaines intègrent la notion de dérive ou de consanguinité comme MacNeil et al (1984) chez la caille japonaise, d'autres prennent en compte l'effet Bulmer comme Sorensen et Hill (1982) chez la drosophile ou Kennedy (1984) chez la vache laitière; aucune à notre connaissance n'a soulevé la question de l'homogamie. 


\section{RÉFÉRENCES}

Anonyme (1950-1968) Chroniques du turf. Établissement Chéri Éditeur, Paris

Anonyme (1969-1982) Abrégé des courses au galop. Union nationale interprofessionnelle du cheval, Paris

Bulmer MG (1971) The effect of selection on genetic variability. Amer Nat 105, 201-211

Falconer DS (1981) Introduction to Quantitative Genetics (2nd edition). Longman, Londres

Fisher RA (1918) The correlation between relatives on the supposition of Mendelian inheritance. Trans $R$ Soc Edinburgh 52, 399-433

Fredeen HT (1984) Selection limits: have they been reached with pigs? Can J Anim Sci $64,223-234$

Hintz RL (1977) Genetics of performance in the horse. J Anim Sci 51, 583-594

Hunton P (1984) Selection limits: have they been reached in the poultry industry? Can $J$ Anim Sci 64, 217-221

Kennedy BW (1984) Selection limits: have they been reached with the dairy cow? Can $J$ Anim Sci 64, 207-215

Langlois B (1975) Analyse statistique et génétique des gains des pur-sang anglais de trois ans dans les courses plates françaises. Ann Génét Sél Anim 7, 387-408

Langlois B (1980) Heritability of racing ability in thoroughbreds - a review. Livest Prod Sci 51, 583-594

Langlois B (1981) Proposition d'extension du modèle génétique additif classique à des situations non panmictiques. Ann Génét Sél Anim 13, 151-164

Langlois B (1990) Incidence de la sélection et de l'homogamie sur les paramètres du modèle génétique additif. Genet Sel Evol 22, 119-132

Lush JL (1945) Animal Breeding Plans. Iowa University Press

MacNeil MD, Kress DD, Flower AE, Webb RP, Blackwell RL (1984) Effects of mating system in Japanese quail. Theor Appl Genet 67, 403-412

Mirande SL, Van Vleck LD (1985) Trends in genetic and phenotypic variances for milk production. J Dairy Sci 68, 2278-2286

Moureaux S (1994) Étude de la variabilité génétique des principales races chevalines françaises sur la base de l'information généalogique et du polymorphisme biochimique. Mémoire Ina-Paris-Grignon

Moureaux S, Verrier É, Ricard A, Mériaux JC (1996) Genetic variability within French race and riding horse breeds from genealogical data and blood marker polymorphisms. Genet Sel Evol 28, 83-102

Sorensen DA, Hill WG (1982) Effect of short term directional selection on genetic variability: experiments with Drosophila melanogaster. Heredity 48, 27-33

Tavernier A (1988) Advantages of BLUP animal model for breeding value estimation in horses. Livest Prod Sci 7, 591-605

Tolley EA, Notter DR, Marlowe TJ (1985) A review of the inheritance of racing performance in horses. Anim Breed Abstr 53, 163-185

Verrier É, Colleau JJ, Foulley JL (1991) Methods for predicting response to selection in small populations under additive genetic models: a review. Livest Prod Sci 29, 93-114

Wilson SP, Goodale HD, Kyle WH, Godfrey EF (1971) Long term selection for body weight in mice. $J$ Heredity $62,228-234$

Yoo BH (1980) Long term selection for a quantitative character in large replicate populations of Drosophila melanogaster. Genet Res Camb 35, 1-17 